\title{
EXPULSIVE HEMORRHAGE AFTER GLAUCOMA FILTERING SURGERY
}

\author{
Nada Jiraskova*, Pavel Rozsival, Jana Pozlerova, Marie Ludvikova, Mariya Burova
}

\author{
Department of Ophthalmology, University Hospital, Hradec Kralove, Czech Republic \\ e-mail: jirasnad@fnhk.cz
}

Received: January 28, 2009; Accepted: August 17, 2009

Key words: Suprachoroidal hemorrhage/Glaucoma filtering surgery/Outcomes

Aims: To evaluate the incidence, patient risk factors, diagnosis and management of suprachoroidal hemorrhage $(\mathrm{SCH})$ after glaucoma filtering surgery.

Methods: Retrospective case series study comprised 1553 eyes having glaucoma filtering surgery during the last 15 years (between January 1993 and December 2007) at our department. Observations included incidence, patient risk factors, peri and postoperative diagnosis, management, and outcomes of $\mathrm{SCH}$ after this procedure.

Results: Two cases of SCH were revealed. In one eye SCH developed at the end of surgery, in the other eye in the postoperative period. Both patients had systemic and ocular risk factors (hypertension, high preoperative intraocular pressure, myopia, pseudoexfoliation, aphakia or pseudophakia). Visual functions improved in the case of intraoperative SCH over 2 months withouth surgical intervention, in the eye with delayed postoperative SCH visual outcome was poor despite multiple lavages of anterior chamber and vitreous cavity.

Conclusion: Suprachoroidal hemorrhage, both expulsive and delayed, is a rare, but severely debilitating complication of glaucoma surgery.

\section{INTRODUCTION}

Suprachoroidal hemorrhage ( $\mathrm{SCH})$ is a much feared complication of all types of intraocular surgery. Although rare, it is typically associated with poor long.term prognosis for visual functions. The latter has prompted efforts to better understand the pathogenesis, identify the patients at risk and to improve treatment of this event.

Suprachoroidal hemorrhage is defined as blood within the suprachoroidal space. Classification can be done in several ways: $\mathrm{SCHs}$ may be categorized with respect to size and extent of hemorrhage, by their relationship to intraocular surgery, and also by precipitating events ${ }^{1}$. When categorized with respect to size, $\mathrm{SCHs}$ can vary from a small area of involvement to massive hemorrhage into the suprachoroidal space. Suprachoroidal hemorrhage has been reported to occur in the setting of all types of intraocular procedures, including cataract extraction ${ }^{2,3}$, penetrating keratoplasty ${ }^{4,5}$, glaucoma filtering surgery ${ }^{6.9}$ and vitreoretinal surgery ${ }^{10,11}$. Timing of development of $\mathrm{SCH}$ in relation to intraocular surgery is another method of classifying the condition - it may develop at the time of surgery or in the postoperative period. Finally, $\mathrm{SCHs}$ can be categorized by precipitating events, in particular, either penetrating or blunt trauma ${ }^{1}$.

Studies examining the risk of SCH in relation to glaucoma filtering surgery must be separated according to type of SCH. The incidence of expulsive SCH during glaucoma surgery has been reported to be approximately $0.15 \%\left(\mathrm{ref}^{9}\right)$. Delayed SCH after glaucoma surgery is believed to be precipitated by prolonged postoperative hypotony and inflammation ${ }^{1}$ and its incidence is approximately 10 -fold greater than that of perioperative $e^{6-8}$.

\section{MATERIALS AND METHODS}

Retrospective case series study comprised 1553 eyes had undergone glaucoma filtering surgery during the last 15 years (between January 1993 and December 2007) at our department. Observations included incidence, patient risk factors, peri and postoperative diagnosis, management, and outcomes of SCH after this procedure.

\section{RESULTS}

Two cases of SCH were revealed among 1553 glaucoma filtering surgeries. Hence the incidence of this serious complications was $0.13 \%$.

\section{Case 1}

A 51-year-old man was followed up and treated for many years for multiple serious ocular diseases. From childhood he had suffered from progressive degenerative myopia bilaterally. At the age of 18 he developed retinal detachment in his right eye and uderwent unsuccessful operation, 1 year later glaucoma surgery was performed and 8 years later the blind right eye was enucleated for painful glaucoma. At that time elevated intraocular pressure (IOP) in his left eye was revealed and the patient was treated by timolol $0.5 \% \mathrm{gtt}$. Owing to subcompensation of glaucoma and progression of complicated cataract he was referred to our department. Uncomplicated phacoemulsification without intraocular lens (IOL) implantation was performed in 1995. Best corrected visual acuity (BCVA) improved to 0.3 , and relative and absolute scotomas caused by both glaucoma and degenerative myopia were detected on visual field examination. During the 
next 7 years glaucoma remained subcompensated despited maximal medical treatment (timolol, latanoprost, brinzolamid) and selective laser trabeculoplasty (SLT), that was performed in 1999. In 2002 he was again referred to our department for glaucoma surgery. BCVA of his left eye was 0.25 , his visual field was almost completely lost with a small residual island of vision remaining and IOP varied from 17 to $26 \mathrm{mmHg}$. Slit lamp examination revealed a quiet anterior chamber, aphakia, and minor fibrosis of posterior capsule. On fundus examination large chorioretinal atrophy around the optic nerve head and at the posterior pole with defects in Bruch's membrane were found. The cup to disc (C/D) ratio was 0.9 and there were small hemorrhages on the disc margin. Systemically the patient was treated for hypertension. Althouth we were aware of multiple risk factors in this case, we decided to provide glaucoma filtering surgery as a last chance for the patient to save the remaining visual functions. Uncomplicated trabeculectomy with mitomycin $\mathrm{C}$ was performed. On routine postoperative examination 6 hours after surgery good filtering bleb, quiet, deep, and clear anterior chamber were observed. In the evening (10 hours after operation) the patient developed nausea, paresthesia, and chestpain. His blood pressure was $160 / 95 \mathrm{mmHg}$. He was examined by both a neurologist and an internist, and systemic sedative and analgesic therapy was administered with good effect. Two hours later he reported no any complaints and the status of his left eye remained unchanged. In the morning on the first postoperative day he had vision of light perception with inaccurate projection of light in 3 quadrants, IOP was $8 \mathrm{mmHg}$. Examination showed lid edema, chemosis of conjunctiva, clear cornea, $3 \mathrm{~mm}$ hyphaema, no signs of iris incarceration in the trabeculectomy ostion, well centered pupil, aphakia and massive vitreous hemorrhage. An ultrasound examination confirmed the diagnosis SCH (Fig. 1). Anti-glaucoma medication was discontinued, and the patient was started on topical and systemic antibiotics and steroids and compressive patching was ap-

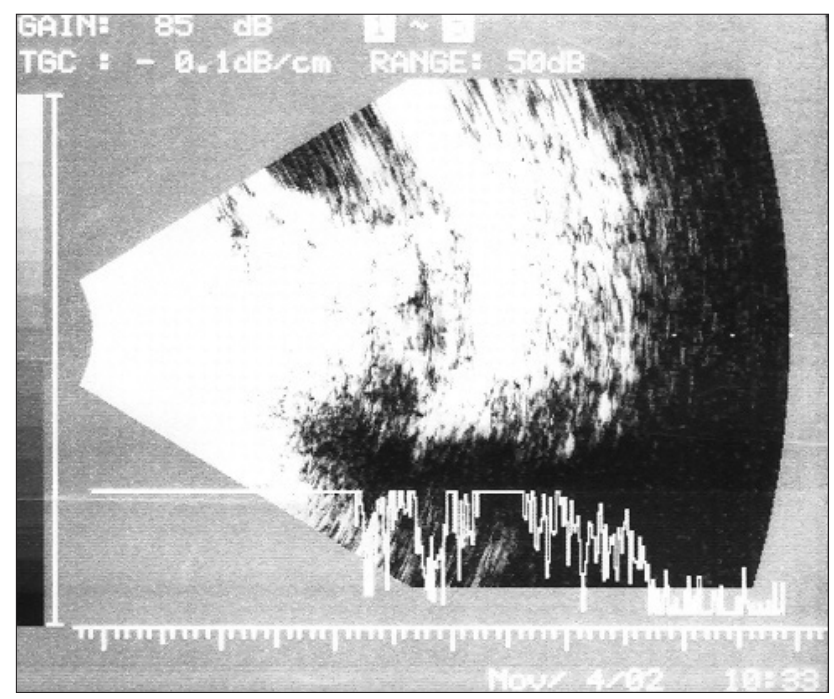

Fig. 1. B-scan ultrasonography showing hemorrhagic choroidal detachment. plied. At noon the IOP was $12 \mathrm{~mm} \mathrm{Hg}$ and in the evening $16 \mathrm{~mm} \mathrm{Hg}$. A vitreoretinal surgeon was consulted and did not recommend performing a vitreoretinal procedure. After 6 days the patient was scheduled for lavage of the anterior chamber. Two limbal incision were made and blood was removed from the anterior chamber. Dark red blood gushed out from the vitreous space and was also removed. Postoperatively BCVA was light perception with inaccurate projection of light in 3 quadrants, IOP was $15 \mathrm{mmHg}$, and hyphaema reappeared. Lavage of the anterior chamber was performed again 12, 15 and 20 days after glaucoma surgery. Systemic steroids were gradually discontinued. Hyphaema and vitreous hemorrhage slowly disappeared during the next 2 months. Vision remained on light perception with inaccurate projection, and IOP was well controlled.

\section{Case 2}

A 74-year-old woman who had bilateral asymetric pseudoexfoliative glaucoma (right eye $\mathrm{C} / \mathrm{D}$ ratio 0.3 , and minimal visual field changes; left eye $\mathrm{C} / \mathrm{D}$ ratio 0.7 , and wide Bjerrum's upper and narrow lower scotomas) underwent bilateral phacoemulsification with hydrophilic acrylic IOLs implantation in 2002. For the next four years glaucoma was satisfactorily managed by topical betaxolol and latanoprost. At the beginning of 2007 rapid increase of the IOP of the left eye was found and maximal medical therapy failed. She was referred to our department. BCVA was 0.4 in the right eye and 0.3 in the left eye, IOP was $20 \mathrm{mmHg}$ in the right eye and $55 \mathrm{mmHg}$ in the left eye, there was slight progression in visual field defects of the left eye. Systemically the patient was treated for hypertension. She was scheduled for glaucoma filtering surgery. Conjunctival limbal-based flap, partial-thickness scleral flap, goniotomy, trabeculectomy, and iridectomy were performed. At the time of closing the scleral flap expulsive hemorrhage withouth prolaps of intraocular contains was observed. The wound was tighthly closed with 2 nylon 10-0 sutures, and covered with conjunctiva. No attempt was made to drain the suprachoidal blood. The patient did not complain of any pain. On the first

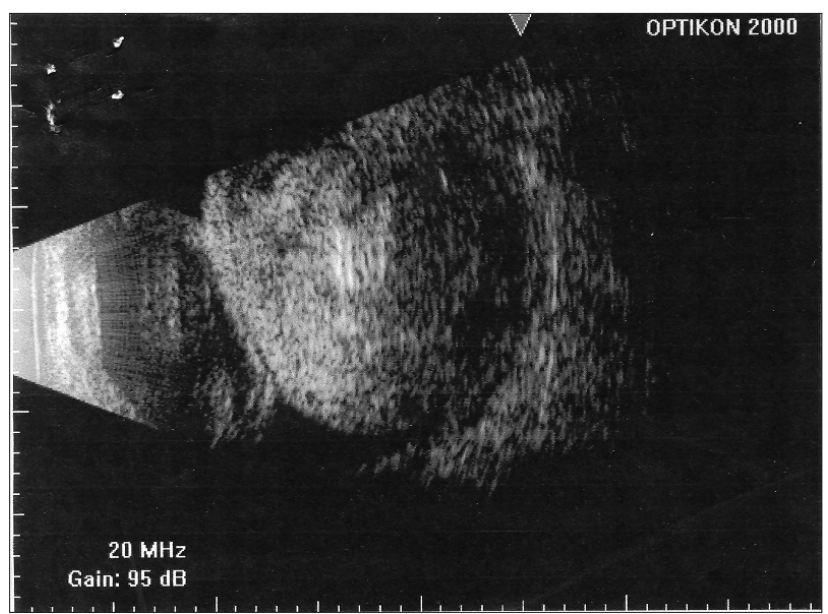

Fig. 2. B-scan echogram demonstrating a massive $\mathrm{SCH}$. 
postoperative day vision was light perception with inaccurate projection of light in 2 quadrants, IOP was $10 \mathrm{mmHg}$. Slit-lamp examination showed normally configurated filtering bleb, clear cornea, a shallow anterior chamber, no signs of iris incarceration in the trabeculectomy ostion, wide patent iridectomy, well centered pupil, IOL retropupilary, pseudoexfoliation, posterior capsule fibrosis, and massive vitreous hemorrhage. An ultrasound examination confirmed the diagnosis of SCH (Fig. 2). Topical antibiotics, steroids, and mydriatics were started. A vitreoretinal specialist was consulted and did not recommend performing vitreoretinal surgery. The patient was regulary followed-up, the SCH slowly disappeared, and BCVA improved over two months to 0,3 . The IOP remained stable (10-12 $\mathrm{mm} \mathrm{Hg}$ ) withouth medication.

\section{DISCUSSION}

Several theories have been postulated to explain the mechanism whereby SCHs develop ${ }^{12,13}$. Hypotony appears to be the major precipitating factor resulting in rupture of a necrotic long or short posterior ciliary artery. Retrospective studies and case reports indicate that certain sytemic and/or ocular risk factors are associated with the development of $\mathrm{SCH}$, both during and after surgery. In particular, sclerosis and fragility of choroidal vessels associated with advanced age, systemic hypertension, and arteriosclerosis have been frequently described as predisposing factors. Various ocular conditions bave been reported to be associated with $\mathrm{SCH}$, including glaucoma, elevated IOP, aphakia, axial myopia, and inflammation. The mechanism by which these ocular risk factors are believed to have an impact on development of $\mathrm{SCH}$ is similar. These ocular conditions are presumed to weaken the integrity of the long posterior ciliary arteries by promoting vascular necrosis. This, in turn, would make these vessels more susceptible to rupture. In the case of surgical aphakia, the absence of the lens and zonular support is believed to allow more stretching and separation of the uvea from sclera during ciliochoroidal effusions. Loss of scleral rigidity and/or choroidal vascular fragility are also believed to be responsible for the association between $\mathrm{SCH}$ and axial myopia ${ }^{1}$. Pseudoexfoliation syndrome is not commonly described in association with $\mathrm{SCH}$. We belive that pseudoexfoliation (PEX) should be considered as a risk factor for development of SCH. The association between PEX and glaucoma has been well documented and pseudoexfoliative glaucoma is believed to be secondary open-angle glaucoma which differs from chronic simple glaucoma by manifesting higher IOP, rapid loss of visual field, and poorer response to medication ${ }^{14}$. Although the pathogenesis of PEX remains obscure, depositions of characteristic fibrillar extracelullar material in various ocular and also extraocular tissues have been extensively reported. Occurrence of PEX material in the walls of posterior ciliary artery, extraocular muscles, walls of vortex veins, orbital connective-tissue septa, optic nerve sheaths, and skin and/or visceral organ specimens indicate that the
PEX syndrome is an even more widespread process and possibly a systemic disorder ${ }^{15,16}$.

Coughing, straining, nausea, vomiting, and Valsalvatype maneuvers have all been implicated in precipitating $\mathrm{SCH}$, either from bucking during general anesthesia, or in the postoperative period ${ }^{5,8,17}$. All these maneuvers lead to increased episcleral venous pressure, resulting in an increased pressure gradient across the wall of necrotic ciliary vessels, thereby promoting their rupture ${ }^{1}$. We believe that this was the mechanism of development of $\mathrm{SCH}$ in our first case together with other risk factors.

Controversy still exists regarding the best course of management of SCH. If an expulsive SCH occurs during intraocular surgery, prompt and decisive reaction is neccessary. Suturing of all surgical incisions and closure of the eye allows the IOP to rise to sufficient level to tamponade the bleeding vessels. If intraocular contents are expelled they should be reposited as quickly as possible. This was not observed in our patients. The long-term benefit of performing posterior sclerotomies acutely at the time the $\mathrm{SCH}$ occurs remains debatable. The efficacy of drainage sclerotomies was tested in an experimental model of $\mathrm{SCH}$ in rabbit eyes, where the creation of acute drainage sclerotomies resulted in a further extension of the hemorrhage into the retina and vitreous. The conclusion therefore was that creation of immediate sclerotomies during $\mathrm{SCH}$ is detrimental to eyes ${ }^{18}$. Postoperative management of $\mathrm{SCH}$ depends on clinical features. However, the decision to reoperate on patients with $\mathrm{SCH}$ is still controversial. When reoperation is considered, the surgical approach can be one of two choices: drainage procedures and/or vitreoretinal surgery. A vitreoretinal surgeon should be consulted before the reoperation because of common posterior segment complications such as retinal dialysis and detachment after the drainage of $\mathrm{SCH}$. In our first case, where multiple lavages of anterior chamber and vitreous cavity were performed, the patient lost his vision (but it is necessary to consider that before this operation his visual functions had seriously deteriorated to the point of blindness). In the other patient, who was followed up without surgical intervention, BCVA improved during 2 months to 0.3 .

These two reported patients with SCHs were the only two cases with this complication after glaucoma filtering surgery that were revealed in 1553 glaucoma procedures performed over the last 15 years (between January 1993 and April 2007) at our clinic, which is an incidence of $0.13 \%$ in our hands. There was no difference between the incidence of perioperative and delayed SCH in this series.

A thorough understanding of the pathophysiology, risk factors, and clinical outcome of this entity can help ophthalmic surgeons to avoid this complication in patients at risk and to optimally treat patients in whom SCH develops.

\section{ACKNOWLEDGEMENT}

Supported in part by research project MZO 00179906. 


\section{REFERENCES}

1. Chu T, Green RL. Suprachoroidal Hemorrhage. Surv Ophthalmol 1999; 43:471-486.

2. Eriksson A, Koranyi G, Seregard S, Philipson B. Risk of acute suprachoroidal hemorrhage with phacoemulsification. J Cataract Refract Surg 1998; 24:793-800.

3. Rozsival P, Aschenbrennerova J. Favourable Outcome of Expulsive Haemorrhage During Cataract Surgery by Tunel Incision. Cs Ofta 1994; 50:31-34.

4. Duncker GI, Rochels R. Delayed suprachoroidal hemorrhage after penetrating keratoplasty. Int Ophthalmol 1996;19:173-176.

5. Ingraham JH, Donnenfeld ED, Perry HD. Massive suprachoroidal hemorrhage in penetrating keratoplasty. Am J Ophthalmol 1989;108:670-675.

6. Givens K, Shields MB. Suprachoroidal hemorrhage after glaucoma filtering surgery. Am J Ophthalmol 1987;103:689-694.

7. Paysse E, Lee PP, Lloyd MA, Sidoti PA, Fellenbaum PS, Baerveld G, Minckler DS, Heuer DK. Suprachoroidal hemorrhage after Molteno implantation. J Glaucoma 1996; 5:170-175.

8. Ruderman JM, Harbin TS Jr, Campbell DG. Postoperative suprachoroidal hemorrhage following filtration procedures. Arch Ophthalmol 1986; 104:201-205.

9. Speaker MG, Guerriero PN, Met JA, Coad CT, Berger A, Marmor M. A case-control study of risk factors for intraoperative suprachoroidal expulsive hemorrhage. Ophthalmology 1991; 98:202-210.
10. Lakhanpal V, Schocket SS, Elman MJ, Dogra MR. Intraoperative massive suprachoroidal hemorrhage during pars plana vitrectomy. Ophthalmology 1990; 97:1114-1119.

11. Piper JA, Han DP, Abrams GW, Mieler WF. Perioperative choroidal hemorrhage at pars plana vitrectomy: a case-control study. Ophthalmology 1993; 100:699-704.

12. Manschot WA. The pathology of expulsive hemorrhage. Am J Ophthalmol 1955; 40:15-24.

13. Zauberman H. Expulsive choroidal haemorrhage: an experimental study. Br J Ophthalmol 1982; 66:43-45.

14. Henry JC, Krupin T, Schmitt M, Lauffer J, Miller E, Ewing MQ, et al. Long-term Follow-up of Pseudoexfoliation and the Development of Elevated Intraocular Pressure. Ophthalmology 1987; 94:545-552.

15. Schlötzer-Schrehardt UM, Koca MR, Naumann GOH. Pseudoexfoliation Syndrome. Arch Ophthalmol 1992; 110:17521756.

16. Streeten BW, Li ZY, Wallace RN, Eagle RC, Keshgegian AA Pseudoexfoliative Fibrillopathy in Visceral Organs of a Patient With Pseudoexfoliation Syndrome. Arch Ophthalmol 1992; 110:1757-1762.

17. Frenkel RE, Shin DH. Prevention and management of delayed suprachoroidal hemorrhage after filtration surgery. Arch Ophthalomol 1986; 104:1459-1463.

18. Lakhanpal V. Experimental and clinical observations on massive suprachoroidal hemorrhage. Trans Am Ophthalmol Soc 1993; 91:545-562. 